\title{
Broadband Rotary Joint Concept for High-Power Radar Applications
}

\author{
Daniel Haas ${ }^{1} \cdot$ Manfred Thumm $^{1,2} \cdot$ John Jelonnek ${ }^{1,2}$
}

Received: 2 September 2020 / Accepted: 21 December 2020 /Published online: 5 January 2021

(C) The Author(s) 2020

\begin{abstract}
To allow antenna movements in azimuth and elevation in high-power radar applications, rotary joints are essential. They allow the rotation of a transmission line and therefore are important transmission line components. In the present paper, a broadband rotary joint concept for high-power W-band radar applications is proposed. To avoid a twist of the polarization plane of a linearly polarized mode, like $\mathrm{HE}_{11}$, a combination of two broadband polarizer is used. A cross polarization of $X_{\mathrm{pol}} \leq-20 \mathrm{~dB}$ can be achieved within the considered frequency range from $90 \mathrm{GHz}$ to $100 \mathrm{GHz}$. This corresponds to a suitable value for radar applications.
\end{abstract}

Keywords High-power microwave $\cdot$ Corrugated waveguide $\cdot$ Transmission line

\section{Introduction}

As already predicted in the late 1970s [1], space debris becomes a major issue for use of space $[2,3]$. In particular, the amount of space debris in low earth orbit (LEO) is increasing rapidly [3]. To detect and map space debris, high-performance radar sensors can be used [3, 4]. Due to the enormous progress in the field of high-power microwave technology, corresponding radar sensors can also be operated in high-frequency bands such as the W-band [5]. Due to the high bandwidth available there, very high resolutions can be achieved $[5,6]$. In the near future, even $\mathrm{W}$-band transmission powers in the range of $100 \mathrm{~kW}$ may be achieved [7]. To realize a Wband radar sensor with such a high transmission power, a suitable high-power amplifier and an appropriate transmission line are required. The transmission line connects the output of the high-power amplifier with the antenna feed. Due to the high power, overmoded transmission

Daniel Haas

daniel.haas@kit.edu

1 IHM, Karlsruhe Institute of Technology (KIT), Kaiserstr. 12, 76131 Karlsruhe, Germany

2 IHE, Karlsruhe Institute of Technology (KIT), Kaiserstr. 12, 76131 Karlsruhe, Germany 
lines are required. A suitable transmission mode is the $\mathrm{HE}_{11}$ hybrid mode in a corrugated waveguide. Low ohmic loss and small mode conversion can be achieved [8, 9].

In principle, for a highly overmoded $\mathrm{HE}_{11}$ transmission line, a rotary joint can be realized by a simple transmission line gap. Due to the low $\mathrm{HE}_{11}$-field components at the waveguide walls, no major influences are expected. The $\mathrm{HE}_{11}$ mode power loss through a transmission line gap can be estimated by [10]:

$$
\Delta P_{\mathrm{HE} 11}=0.39 \cdot\left(\frac{L \lambda}{2 a^{2}}\right)^{3 / 2}
$$

with $L$ as the gap length, $\lambda$ as the free space wavelength, and $D=2 a$ as the waveguide diameter. The $\mathrm{HE}_{11}$ power loss can be already neglected for $L \lambda / 2 a^{2} \leq 1 / 100$. A commonly utilized standard waveguide diameter for high-power transmission lines is $D=63.5 \mathrm{~mm}$. With a wavelength of $\lambda=3 \mathrm{~mm}$ follows: $L \leq 6.7 \mathrm{~mm}$. For a technical realization, this is noncritical. However, by rotation of such a rotary joint, the polarization plane of a linearly polarized mode, like $\mathrm{HE}_{11}$, is twisted. To avoid such issues, the circularly polarized $\mathrm{HE}_{11}$ mode can be used for transmission. Owing to the circular symmetry, no twist of the polarization plane occurs.

To change the polarization of the $\mathrm{HE}_{11}$ mode, suitable broadband polarizers are required: $\mathrm{A}$ first polarizer converts the incident linearly polarized $\mathrm{HE}_{11}$ mode to a circularly polarized wave, and a second polarizer restores the linearly polarized $\mathrm{HE}_{11}$ mode after the transmission line gap. This method provides a simple broadband rotary joint concept for high-power radar applications. Following the reachable cross polarization for such a rotary joint concept shall be investigated.

The paper is organized as follows: Section 2 starts with a suitable polarizer design and discusses possible polarization errors. In Section 3, simple formulas to calculate the cross polarization of the proposed rotary joint concept are derived. Section 4 addresses the issue of mode conversion due to diffraction. Section 5 , the conclusions, closes with a summary of the considered aspects and the proposed rotary joint concept.

\section{Polarizer}

A linearly polarized wave can be polarized circularly by introducing a $90^{\circ}$ phase shift of orthogonal field components [11]. For high-power microwave applications, reflection grids [12] are widely used: Electrical field components parallel to the grid are reflected at the surface of the grid; electrical field components perpendicular to the grid penetrate into the grid and are reflected at the bottom. The resulting time delay can be used for polarization tailoring. Figure 1 shows a rectangular reflection grid with the grid height $h$, the grid period $p$, and the grid width $a$.

In the ideal case, an exact phase shift by $90^{\circ}$ and an amplitude balance of orthogonal field components by $0 \mathrm{~dB}$ can be achieved, within the considered frequency range. At a real polarizer, a phase error $\Delta \varphi$ and an amplitude error $\Delta A$ occur. In general, a frequencydependent elliptical polarization results:

$$
\begin{gathered}
E_{\mathrm{x}}=\Delta A \cdot \cos (\omega t+\Delta \varphi) \\
E_{\mathrm{y}}=\sin (\omega t)
\end{gathered}
$$

In the ideal case (purely circular polarization) applies: $\Delta \varphi=0$ and $\Delta A=1$. The design of a suitable polarization grid for high-power W-band radar applications is discussed in detail in 
Fig. 1 Rectangular reflection grid with the grid height $h$, the grid width $a$, and the grid period $p$ (photo from [13])

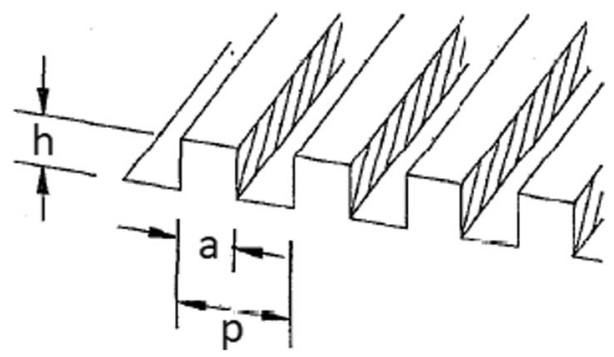

[13]: Good broadband frequency behavior can be achieved for a grid width $a=0.25 \mathrm{~mm}$, a grid period $p=1 \mathrm{~mm}$, and a grid height $h=0.48 \mathrm{~mm}$. To avoid critical field strengths and electrical arc breakdown, curvature radii at the upper end of the grid are used $(R=0.1 \mathrm{~mm}$ [13]). Figure 2 shows the corresponding phase error $\Delta \varphi$ (in blue, solid) and amplitude error $\Delta A$ (in brown, solid) for the grid parameters introduced above, within the frequency range from $90 \mathrm{GHz}$ to $100 \mathrm{GHz}$ [13]. Note that the amplitude error $\Delta A$ is scaled in decibels. Figure 2 shows that the phase error can be limited to $\Delta \varphi \leq \pm 5.8^{\circ}$. The small variation of the amplitude error $\Delta A$ is a result of numerical uncertainties [13] and can be neglected. For comparison, also results of a less suitable parameter combination are shown $(a=1.06 \mathrm{~mm}, p=1.75 \mathrm{~mm}$, and $h=0.42 \mathrm{~mm}$, dashed curves [13]). The polarization errors are much larger in this case.

\section{Cross Polarization}

In the following, the cross polarization of the proposed rotary joint concept is derived. For simplification, a plane wave approximation is used. For a waveguide diameter $D \geq 12 \lambda$, the

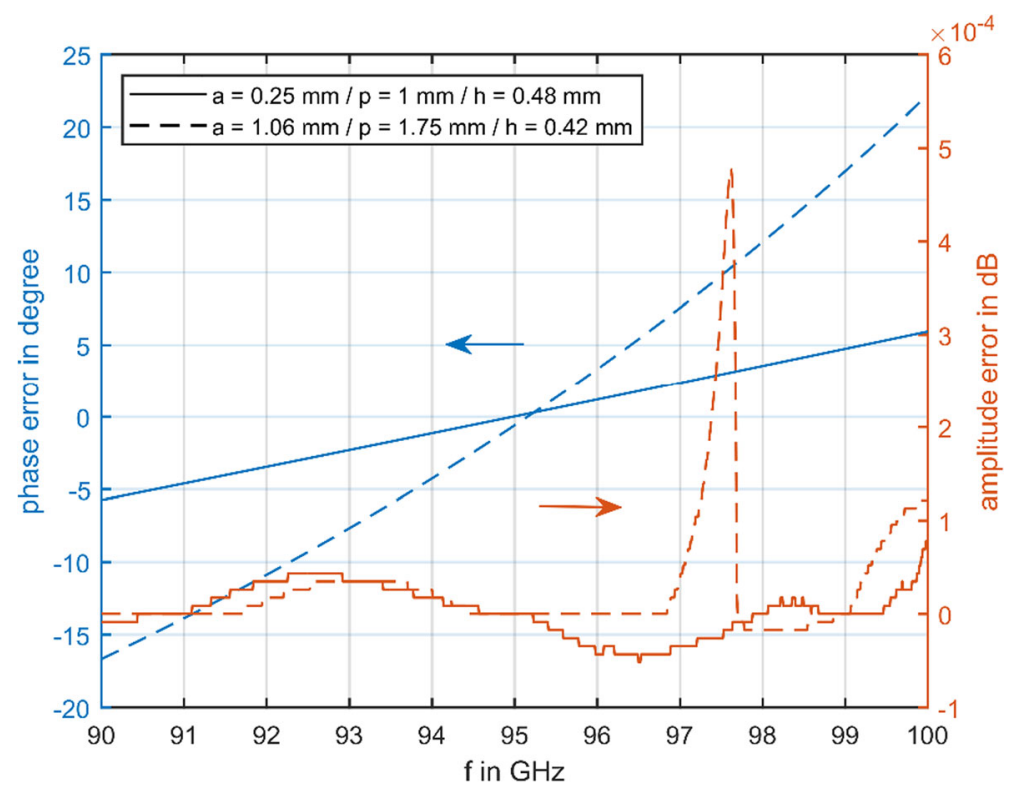

Fig. 2 Phase error $\Delta \varphi$ and amplitude error $\Delta A$ of a rectangular phase grid with suitable (solid curves) and less suitable (dashed curves) parameter combination [13]. The grid width $a$, the grid period $p$, and the grid height $h$ are defined in Fig. 1 
results of a plane wave approximation can be transferred to a $\mathrm{HE}_{11}$ wave [12]. Two identical polarizers are used. Figure 3 shows a corresponding CAD (computer-aided design) model, which will be used for future full wave simulations.

\subsection{Principle Approach}

As input, an ideal $45^{\circ}$ linearly polarized plane wave with unit amplitude is assumed. The propagation direction shall be in positive $z$-direction $\left(E_{z}=0\right)$. Therefore, the other two field components are given by:

$$
\begin{aligned}
& E_{\mathrm{x}}=\sin (\omega t) \\
& E_{\mathrm{y}}=\sin (\omega t)
\end{aligned}
$$

At the first polarizer, the $E_{\mathrm{x}}$ component is delayed by $\varphi_{\mathrm{x}}=\pi / 2+\Delta \varphi$. The present phase error is considered by $\Delta \varphi$. As already mentioned, the amplitude error $\Delta A$ can be neglected. With $\sin (x+\pi / 2)=\cos (x)$ follows:

$$
\begin{gathered}
E_{\mathrm{x}}=\cos (\omega t+\Delta \varphi) \\
E_{\mathrm{y}}=\sin (\omega t)
\end{gathered}
$$

A rotation by $\phi$ of the rotary joint can be described by the rotation matrix:

$$
\left(\begin{array}{c}
E_{\mathrm{x}}^{\prime} \\
E_{\mathrm{y}}^{\prime \prime}
\end{array}\right)=\left(\begin{array}{cc}
\cos (\phi) & \sin (\phi) \\
-\sin (\phi) & \cos (\phi)
\end{array}\right) \cdot\left(\begin{array}{c}
E_{\mathrm{x}} \\
E_{\mathrm{y}}
\end{array}\right)
$$

With Eq. (4) follows:

$$
\left(\begin{array}{l}
E_{\mathrm{x}}^{\prime} \\
E_{\mathrm{y}}^{\prime}
\end{array}\right)=\left(\begin{array}{cc}
\cos (\omega t+\Delta \varphi) & \sin (\omega t) \\
\sin (\omega t) & -\cos (\omega t+\Delta \varphi)
\end{array}\right) \cdot\left(\begin{array}{c}
\cos (\phi) \\
\sin (\phi)
\end{array}\right)
$$

At the second polarizer the $E_{\mathrm{y}}^{\prime}$ component is delayed by $\varphi_{\mathrm{y}}=\pi / 2+\Delta \varphi$. With $\sin (x+\pi / 2)=$ $\cos (x)$ and $\cos (x+\pi / 2)=-\sin (x)$ follows:

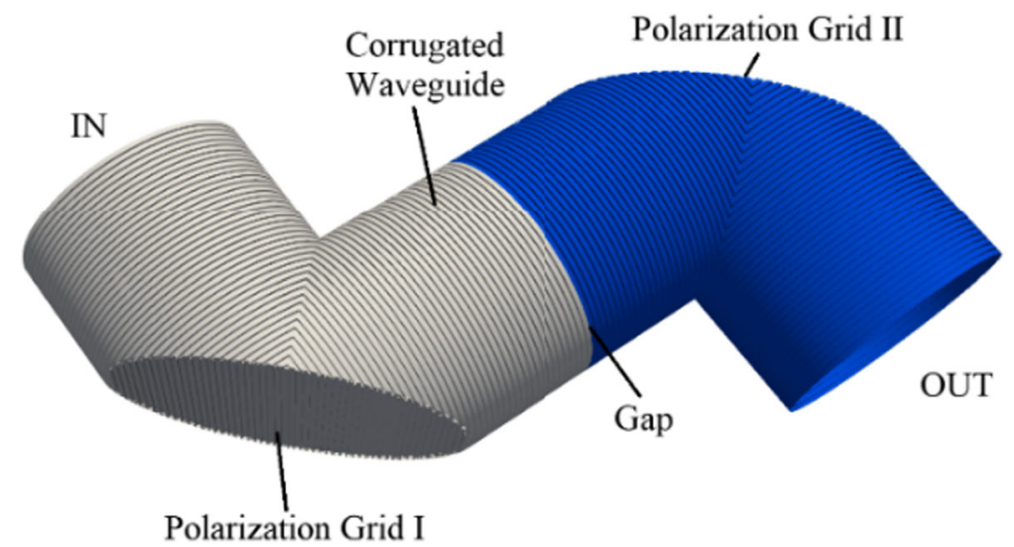

Fig. $3 \mathrm{CAD}$ model of the rotary joint, with polarizer grids discussed in the text and corrugated waveguides with an effective corrugation depth of $\lambda / 4$ 


$$
\left(\begin{array}{c}
E_{\mathrm{x}}^{\prime} \\
E_{\mathrm{y}}^{\prime}
\end{array}\right)=\left(\begin{array}{cc}
\cos (\omega t+\Delta \varphi) & \sin (\omega t) \\
\cos (\omega t+\Delta \varphi) & \sin (\omega t+2 \Delta \varphi)
\end{array}\right) \cdot\left(\begin{array}{c}
\cos (\phi) \\
\sin (\phi)
\end{array}\right)
$$

The cross polarization is defined as the ratio of the time averaged signal power in the desired polarization plane and the time averaged signal power in the orthogonal polarization plane. Equation (7) describes a $45^{\circ}$ linearly polarized wave. To separate the orthogonal polarization planes, an appropriate rotation matrix is used. This rotates the coordinate system by $45^{\circ}$ :

$$
\left(\begin{array}{l}
E_{\mathrm{x}}^{\prime \prime} \\
E_{\mathrm{y}}^{\prime \prime}
\end{array}\right)=\left(\begin{array}{cc}
\cos (\pi / 4) & \sin (\pi / 4) \\
-\sin (\pi / 4) & \cos (\pi / 4)
\end{array}\right) \cdot\left(\begin{array}{c}
E_{\mathrm{x}}^{\prime} \\
E_{\mathrm{y}}^{\prime}
\end{array}\right)
$$

The cross polarization follows as:

$$
X_{\mathrm{pol}}=\frac{\left\langle E_{\mathrm{y}}^{\prime \prime}, E_{\mathrm{y}}^{\prime \prime}\right\rangle}{\left\langle E_{\mathrm{x}}^{\prime \prime}, E_{\mathrm{x}}^{\prime \prime}\right\rangle}
$$

The inner product $\langle x, y\rangle$ is defined as [14]:

$$
\langle x, y\rangle=\lim _{T \rightarrow \infty} \frac{1}{T} \int_{-T / 2}^{+T / 2} x \cdot y^{*} \mathrm{~d} t
$$

Using a small-angle approximation, the introduced equations lead to the cross polarization:

$$
X_{\mathrm{pol}} \approx \frac{\Delta \varphi^{2}}{1+\Delta \varphi^{2}} \cdot \sin ^{2}(\phi)
$$

\subsection{Results}

Figure 4 shows the numerically calculated cross polarization, using Eqs. (7), (8), and (9) without small-angle approximation. The polarization errors introduced in Section 2 are used (see Fig. 2, solid lines). The amplitude error $\Delta A$ is neglected. Figure 4 shows that just for $\phi=180^{\circ}$ the cross polarization is frequency independent. This is related to constructive/destructive superposition of the single polarization errors of the first and the second polarizer. However, the cross polarization is always below $20 \mathrm{~dB}$. This corresponds to a suitable value for radar applications [15]. Furthermore, the worst-case value of $X_{\text {pol }} \approx-20 \mathrm{~dB}$ just occurs at the frequency band edges at $90 \mathrm{GHz}$ and $100 \mathrm{GHz}$. Within the frequency range, the cross polarization is even better. Comparison of the results shown in Fig. 4 and the approximation derived in Eq. (12) shows good agreement.

The rotary joint concept will be experimentally proven later, when the whole transmission line will be manufactured.

\section{Mode Conversion}

In the utilized polarizer arrangement, mode conversion due to diffraction occurs $[10,16]$. The amount of mode conversion loss of the $\mathrm{HE}_{11}$ mode can be estimated with Eq. (1) and $L=D$ [10]. For radar applications, also the exact mode content is important. Spurious modes could 


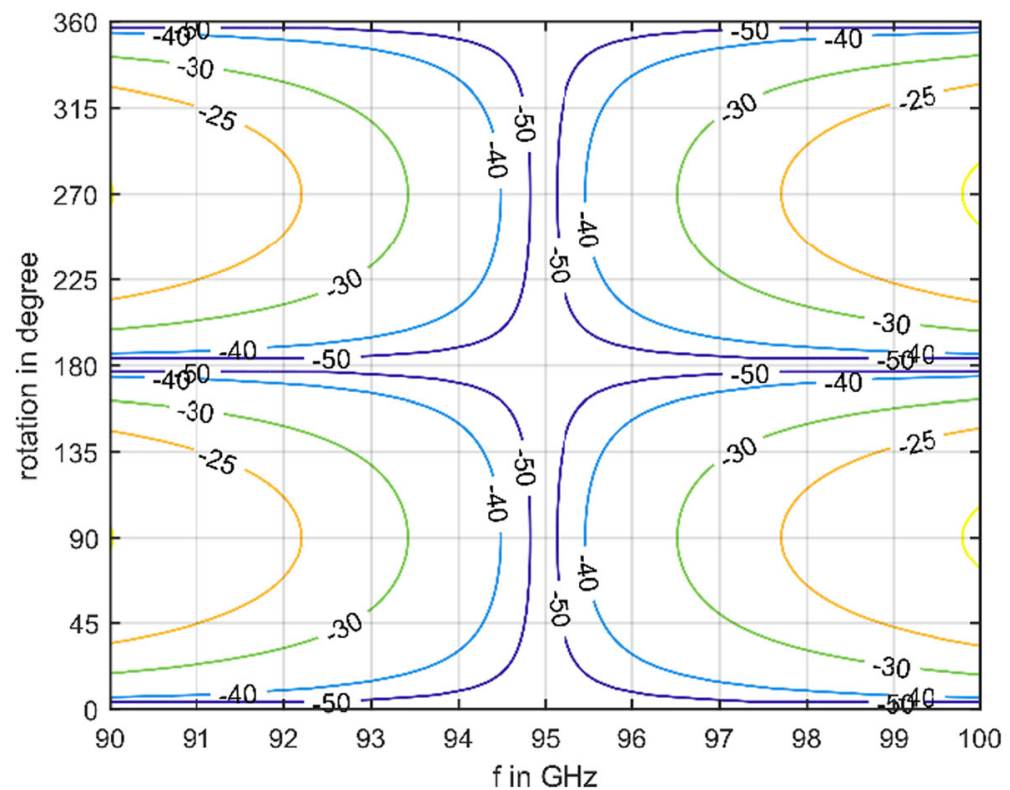

Fig. 4 Cross polarization in decibels as function of the frequency $f$ and the rotation angle $\phi$ of the rotary joint, with suitably designed polarizers

impair the cross polarization and the sidelobe level of the radar antenna. For a highperformance radar sensor, these effects have to be taken into account.

To calculate the mode conversion at a miter bend, the gap theory is commonly employed $[10,16,17]$. The miter bend is represented as a gap with the gap length $L=D$. To determine the electric/magnetic field components at the output plane, the radiated field components from the input plane are calculated, at the location of the output plane. The field components at the position $\vec{r}$, radiated from an arbitrary aperture $A$, can be calculated for $k \backslash$ cdot $R \gg 1$ by [18]:

$$
\begin{aligned}
& \vec{E}=j k \int_{A}\left[\left(\overrightarrow{e_{R}} \times \vec{M}\right)+Z \cdot \overrightarrow{e_{R}} \times\left(\overrightarrow{e_{R}} \times \vec{J}\right)\right] \cdot G \mathrm{~d} \vec{A} \\
& \vec{H}=-j \frac{k}{Z} \int_{A}\left[Z \cdot\left(\overrightarrow{e_{R}} \times \vec{J}\right)-\overrightarrow{e_{R}} \times\left(\overrightarrow{e_{R}} \times \vec{M}\right)\right] \cdot G \mathrm{~d} \vec{A}
\end{aligned}
$$

with:

$$
\begin{gathered}
G=\frac{e^{-j k\left|\overrightarrow{r_{0}}-\vec{r}\right|}}{\left|\overrightarrow{r_{0}}-\vec{r}\right|} \\
\overrightarrow{e_{R}}=\frac{\vec{R}}{R}=\frac{\overrightarrow{r_{0}}-\vec{r}}{\left|\overrightarrow{r_{0}}-\vec{r}\right|}
\end{gathered}
$$

$k=2 \pi / \lambda, Z=120 \pi \Omega$ and the equivalent electric/magnetic current densities [11, 18]:

$$
\begin{aligned}
\vec{J} & =\vec{n} \times \overrightarrow{H_{0}} \\
\vec{M} & =-\vec{n} \times \overrightarrow{E_{0}}
\end{aligned}
$$

The vector $\overrightarrow{r_{0}}$ represents a point at the radiating aperture $A, \overrightarrow{E_{0}} / \overrightarrow{H_{0}}$ the electric/magnetic field components at the aperture, and $\vec{n}$ the normal vector orthogonal to the aperture. 
The assumption $k \backslash$ cdot $R \gg 1$ implied that the distance $R=\left|\overrightarrow{r_{0}}-\vec{r}\right|$ is larger than $\approx 2 \lambda[18]$. This does not imply that Eq. (12) is limited to the far field condition $R>2 D^{2} / \lambda[11]$ with $D$ as the largest aperture dimension.

In $[10,17]$, analytical approaches are used to calculate the $\mathrm{HE}_{11}$-mode power loss with good results. However, simplifications are used which impair the accuracy at high-order modes [10]. For radar applications, the $\mathrm{HE}_{11}$-mode power loss and the exact mode content are important. Therefore, Eq. (12) is solved numerically. With the MATLAB Parallel Computing Toolbox, the required computing time can be still limited to a few minutes.

To determine the mode content at the output plane, the electric field components are interpreted as the superposition of the normal modes within the corrugated waveguide [19]:

$$
\vec{E}=\sum_{n}\left(\vartheta_{n}^{+}+\vartheta_{n}^{-}\right) \cdot \overrightarrow{e_{n}}
$$

with $\vartheta_{n}^{+/-}$as the complex mode amplitude in the forward/backward direction and $\overrightarrow{e_{n}}$ as the electric field components of the $n$th normal mode.

Equation (15) is multiplied by the conjugated transverse electric field components $\vec{e}_{\perp m}$ of the $m$ th normal mode and then integrated in respect of the waveguide cross section:

$$
\int_{A}\left({\overrightarrow{E_{\perp}}}_{e^{\prime}}^{*}\right) \mathrm{d} \vec{A}=\sum_{n}\left(\vartheta_{n}^{+}+\vartheta_{n}^{-}\right) \int_{A}\left(\vec{e}_{\perp n} \cdot \vec{e}_{\perp m}^{*}\right) \mathrm{d} \vec{A}
$$

In consideration of the power orthogonality condition $\int_{A}\left(\vec{e}_{\perp n} \cdot \vec{e}_{\perp m}^{*}\right) \mathrm{d} \vec{A}=0$ for $n \neq m[19$, 20], each summand with $n \neq m$ is vanished. Therefore follows:

$$
\vartheta_{m}^{+}+\vartheta_{m}^{-}=\frac{\int_{A}\left(\vec{E}_{\perp} \cdot \vec{e}_{\perp m}^{*}\right) \mathrm{d} \vec{A}}{\int_{A}\left(\vec{e}_{\perp m} \cdot \vec{e}_{\perp m}^{*}\right) \mathrm{d} \vec{A}}
$$

With $\vartheta_{m}^{-}=0$, just the transversal components of the electric field are required. This can be used to reduce the required computational effort to calculate the field components at the output plane. Due to the circular symmetry, just $\mathrm{HE}_{1 n}$ modes have to be considered for the mode decomposition [10, 17].

As discussed in $[10,16]$, the gap theory neglected mode conversion to high-order modes close to cut-off. However, due to their high ohmic wall losses, these modes are less critical for radar applications, since those will be damped and not radiated from the radar antenna.

Figures 5 and 6 show simulation results for the mode conversion to parasitic low-order modes in dependence of $k a=2 \pi \backslash$ cdot $a / \lambda$. In Fig. 5, the corresponding mode power is plotted and in Fig. 6 the phase relation, relative to the $\mathrm{HE}_{11}$ mode. As predicted from theory $[10,16]$, mode conversion decreases with increasing $\mathrm{ka}$. Both figures show that an appropriate choice of the waveguide diameter is essential. At $f=95 \mathrm{GHz}$, a suitable waveguide diameter is $D=$ $63.5 \mathrm{~mm}(k a \approx 63)$. Mode conversion due to diffraction is less critical here.

In principle, the diffraction losses can be further reduced by choosing a larger diameter. However, above a certain value, mode conversion losses due to alignment tolerances (flange offsets and tilts) of the waveguide segments become dominant. Corresponding calculations are out of scope of the present paper and will be addressed in an upcoming manuscript. 


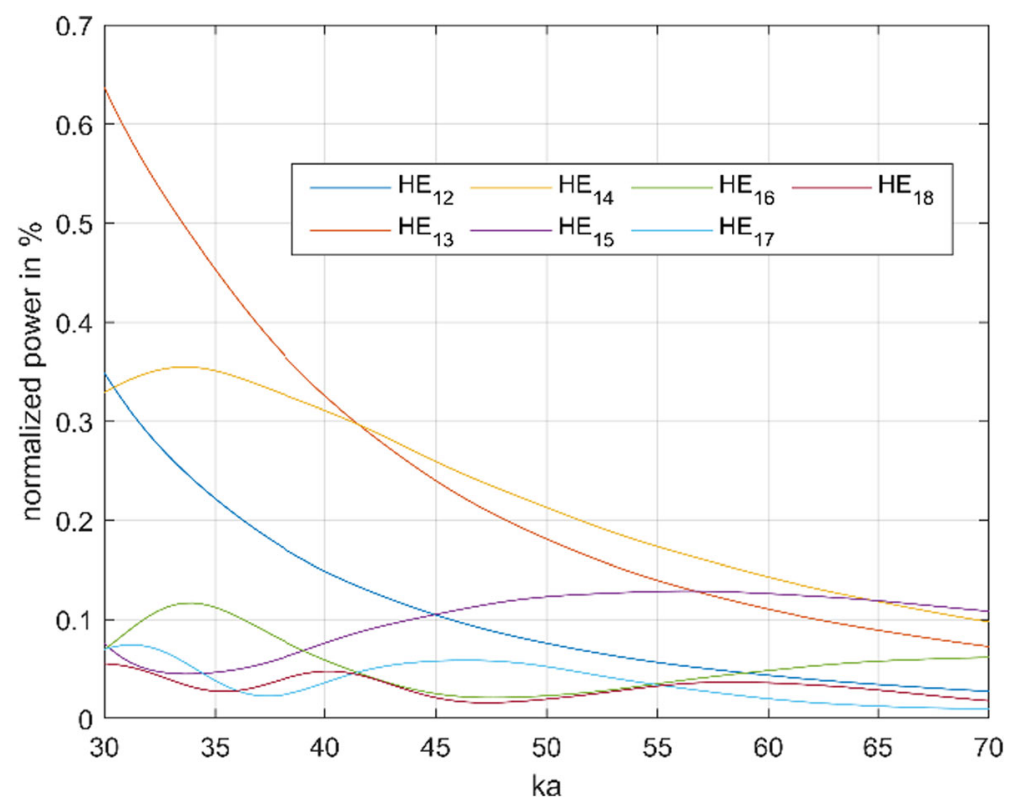

Fig. 5 Power of the parasitic low-order modes

\section{Conclusions}

The present paper addresses a broadband rotary joint concept for high-power W-band radar applications, within the frequency range from $90 \mathrm{GHz}$ to $100 \mathrm{GHz}$. A combination of two broadband polarizers and a simple transmission line gap is proposed as rotary joint. Broadband

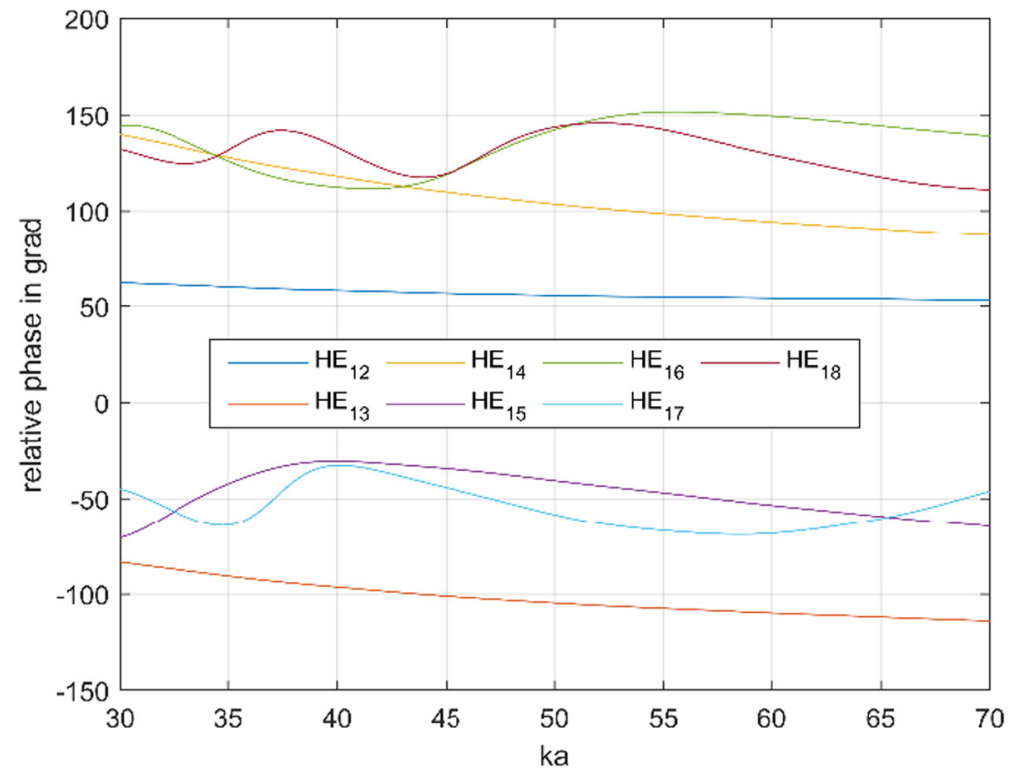

Fig. 6 Phase relation of the parasitic low-order modes, relative to the $\mathrm{HE}_{11}$ mode 
frequency behavior and negligible power loss can be achieved. At the frequency band edges $90 \mathrm{GHz}$ and $100 \mathrm{GHz}$, a worst-case cross polarization of $X_{\mathrm{pol}} \approx-20 \mathrm{~dB}$ occurs. This corresponds to a suitable value for radar applications. Within the whole considered frequency band, the cross polarization is even lower.

For a high-performance radar sensor, spurious modes may be critical, which may impair the cross polarization and the sidelobe level of the radar antenna. At the center frequency of $95 \mathrm{GHz}$, an appropriate waveguide diameter is $D=63.5 \mathrm{~mm}$, for which mode conversion due to diffraction is less critical.

Funding Open Access funding enabled and organized by Projekt DEAL.

Open Access This article is licensed under a Creative Commons Attribution 4.0 International License, which permits use, sharing, adaptation, distribution and reproduction in any medium or format, as long as you give appropriate credit to the original author(s) and the source, provide a link to the Creative Commons licence, and indicate if changes were made. The images or other third party material in this article are included in the article's Creative Commons licence, unless indicated otherwise in a credit line to the material. If material is not included in the article's Creative Commons licence and your intended use is not permitted by statutory regulation or exceeds the permitted use, you will need to obtain permission directly from the copyright holder. To view a copy of this licence, visit http://creativecommons.org/licenses/by/4.0/.

\section{References}

1. D. Kessler and B. Cour-Palais. "Collision Frequency of Artificial Satellites: The Creation of a Debris Belt". Journal of Geophysical Research, Vol. 83, No. A6, 2637-2646 (1978).

2. M. Williamson. "Space Junk makes an Impact", IEE Review, Vol. 52, No. 1, $40-44$ (2006).

3. D. Merholz et al. Detecting, Tracking and Imaging Space Debris, ESA Bulletin, No 109, 128-134 (2002).

4. J. Ender et al. Radar techniques for space situational awareness, International Radar Symposium, 21-26 (2011).

5. M. Czerwinski and J. Usoff. "Development of the Haystack Ultrawideband Satellite Imaging Radar", Lincoln Laboratory Journal, Vol. 21, No. 1, 28-44 (2014).

6. J. Eshbaugh et al. "HUSIR Signal Processing", Lincoln Laboratory Journal, Vol. 21, No. 1, 115-134 (2014).

7. S. Samsonov et al. "Cascade of Two W-Band Helical-Waveguide Gyro-TWTs With High Gain and Output Power: Concept and Modeling", IEEE Transaction on Electron Devices, Vol. 64, No. 3, 1305-1309 (2017).

8. J. Doane. Propagation and Mode Coupling in Corrugated and Smooth-Wall Circular Waveguides, Infrared and Millimeter Waves, Vol. 13, Ch. 5, 123-170 (1985).

9. J. Doane. Design of Circular Corrugated Waveguides to Transmit Millimeter Waves at ITER, Fusion Science and Technology, Vol. 53, No. 1, 159-173 (2008).

10. J. Doane and C. Moeller. "HE11 mitre bends and gaps in a circular corrugated waveguide", International Journal of Electronics, Vol. 77, No. 4, 489-509 (1994).

11. C. Balanis. Antenna Theory: Analysis and Design, Third Edition. Wiley-Interscience, 2005.

12. J. Doane. "Grating Polarizers in Waveguide Miter Bends", International Journal of Infrared and Millimeter Waves, Vol. 13, No. 11, 1727-1743 (1992).

13. D. Haas et al. Broadband Polarizer Miter Bend for High Power Radar Applications, German Microwave Conference, IEEE Xplore, 76-79 (2020).

14. F. Puente and U. Kiencke. Messtechnik: Systemtheorie für Ingenieure und Informatiker. Springer, 2011.

15. R. Touzi, P. Vachon, and J. Wolfe. "Requirement on Antenna Cross-Polarization Isolation for the Operational Use of C-Band SAR Constellations in Maritime Surveillance", IEEE Geoscience and Remote Sensing, Vol. 7, No. 4, 861-865 (2010).

16. B. Katsenelenbaum. "Diffraction on plane mirror in broad waveguide junction". In: Radio Engineering and Electronic Physics 8, 1098-1106 (1963).

17. E. Kowalski. Miter Bend Loss and Higer Order Mode Content Measurements in Overmoded MillimeterWave Transmission Lines. Master-Thesis. Massachusetts Institute of Technology, 2008.

18. K. Kark. Antennen und Strahlungsfelder. Springer Vieweg, 2018.

19. J. Jackson. Classical Electrodynamics. John Wiley \& Sons, 1975. 
20. S. Mahmoud. Electromagnetic Waveguides: Theory and Applications. IET Electromagnetic Waves Series 32, 2006.

Publisher's Note Springer Nature remains neutral with regard to jurisdictional claims in published maps and institutional affiliations. 